\title{
Seguro de Salud y Cobertura de Vacunación en Población Infantil con y sin Experiencia de Desplazamiento Forzado en Colombia
}

\author{
Health insurance for infants and infant vaccination related to forced- \\ displacement in Colombia
}

\author{
Myriam Ruiz-Rodríguez, Lina M. Vera-Cala y Nahyr López-Barbosa
}

Departamento de Salud Pública, Universidad Industrial de Santander-UIS, Colombia.

myriam@uis.edu.co; limavera@uis.edu.co; nlopez@uis.edu.co

Recibido 2 Junio 2007/Enviado para Modificación 18 Noviembre 2007/Aceptado 16 Diciembre 2007

\section{RESUMEN}

Objetivo Determinar la cobertura de vacunación y su relación con el status de afiliación al seguro en niños y niñas menores de cinco años de edad, en condiciones de alta vulnerabilidad social.

Material y métodos Estudio transversal en 514 familias residentes en asentamientos urbanos, receptores de población desplazada por conflicto armado, de 4 municipios del departamento de Santander, Colombia. A través de un muestreo probabilístico con afijación proporcional por municipio se seleccionaron los hogares y se aplicó una encuesta. Los datos de inmunización fueron obtenidos del carné de vacunación. La variable dependiente fue estar vacunado con esquema completo para la edad según los biológicos estipulados por el programa oficial del Ministerio de Protección Social. Mediante regresión logística se modeló la probabilidad de estar vacunado ajustando por las variables sociodemográficas.

Resultados Se estudiaron 369 niños y niñas, de los cuales 48,8 \% pertenecía a familias desplazadas por conflicto armado; $46,1 \%$ de los entrevistados presentaron carné de vacunación. Entre los que tenían carné, contrario a lo esperado, sólo 21,2 \% estaba asegurado y $22,9 \%$ estaba vacunado con esquema completo para la edad. La probabilidad de estar vacunado para la edad, dado que se está afiliado al sistema de salud mediante el régimen subsidiado, es 2,4 veces más, comparada con los no asegurados $(p=0.042)$.

Conclusión Las bajas coberturas de vacunación indican la existencia de barreras de acceso a los servicios y las bajas coberturas de afiliación al seguro sugieren fallas en las políticas de afiliación al seguro para poblaciones que viven en contextos de pobreza y desplazamiento.

Palabras Clave: Seguro de salud, población vulnerable, vacunación, cobertura, Colombia (fuente: DeCS, BIREME). 


\begin{abstract}
Objective Determining vaccination coverage amongst children ( $<5$ years old) having multiple socio-economic risk factors and their relationship to insurance status. Methods This was a cross-sectional study of 514 families from urban settlements receiving people displaced by the armed conflict in 4 municipalities in the Santander department (Colombia). The households were selected by probabilistic sampling, using proportional modelling by municipality. Immunisation data was collected from vaccination cards; interviews provided socio-demographic data. The dependent variable consisted of having the complete vaccination scheme by age according to the official Ministry of Social Protection's programme. The probability of being vaccinated was modelled by a logistical regression, adjusted for sociodemographic variables.

Results 369 children were studied, of whom $48,8 \%$ belonged to families displaced by the armed conflict. $46,1 \%$ of the people being interviewed presented their vaccination cards. Contrary to what had been expected, only $21,2 \%$ of those having a vaccination card were insured and $22,9 \%$ of them had a complete vaccination scheme for their age. The probability of having a complete vaccination scheme for those individuals who were covered by the subsidised health system was 2,4 times higher when compared to those who were not insured $(p=0.042)$.

Conclusions Low vaccination coverage indicated barriers for people living in conditions of poverty and displacement obtaining health services and low insurance coverage suggested faults in health insurance policies addressing similar populations.
\end{abstract}

Key Words: Health insurance, vulnerable population, vaccination, coverage, Colombia (source: MeSH, NLM).

$\mathrm{D}$ esde 1993 Colombia viene disminuyendo el porcentaje de población infantil no asegurada a las instituciones de salud del Sistema General de Seguridad Social en Salud (SGSSS). Con el modelo de aseguramiento de la reforma de 1993 se han logrado importantes metas en cuanto a la equidad en el acceso a los servicios de salud a expensas del incremento de la cobertura de la seguridad en salud a la población más pobre. Sin embargo, estos resultados no han sido tan exitosos para algunos programas específicos como lo es el programa de inmunización infantil, el cual muestra una evidente disminución de las tasas de cobertura después de la reforma (1-6).

Las encuestas de demografía y salud de 1990, 1995, 2000 y 2005 mostraron datos contundentes: el porcentaje de niños y niñas entre 12 y 23 meses de edad que recibieron todas las vacunas disminuyó de 83 en 1990 a 71 en 1995; de 66 en el año 2000 a 58,1 en el año 2005(1,2). Para el año 2001, Prieto y de la Hoz (7) encuentran que en Bogotá previo a la Jornada Nacional de Vacunación, 
solamente 29,1 \% de los niños y niñas menores de dos años estudiados tenían esquema completo de vacunación para la edad, aumentando este porcentaje a 37,9 después de la jornada, y Rojas y Prieto (8) encontraron que a finales del año 2001, después de la Jornada Nacional de Vacunación contra la poliomielitis, sólo 29,8 \% de los municipios del país alcanzó coberturas útiles de vacunación. Una reciente publicación mostró coberturas de vacunación en infantes de 12 a 23 meses de edad inferiores a 70 \% en el año 2003 y las desigualdades en cobertura entre regiones geopolíticas persisten en el país (9).

Estudios previos en el ámbito internacional han demostrado que el uso de servicios preventivos en población infantil asegurada es distinta de la no asegurada $(10,11)$ y que niños y niñas con afiliación a seguros de salud públicos tienen proporciones mayores de cobertura de vacunación que los afiliados a seguros privados (12). Estudios en Colombia han reportado que los niños y niñas afiliados al sistema de seguridad social tienen mayores porcentajes de vacunación que los no afiliados (13), y que el aseguramiento aumenta la probabilidad de usar servicios de vacunación $(3,7)$. En un estudio realizado en Bogotá con población infantil pobre asegurada al régimen subsidiado, Acosta y colaboradores (14) reportaron 22 \% de uso de servicios de vacunación.

El logro de altas coberturas de vacunación para la población infantil es un tema prioritario en los sistemas de salud, pues no solamente es una intervención costo-efectiva que contribuye a disminuir la carga de la enfermedad en la población, sino que el programa de inmunización ofrece mecanismos que permiten en los servicios de salud iniciar una relación con los niños y niñas y las mujeres a quienes tiene que brindar muchas otras intervenciones. En Colombia la reforma del sector salud tuvo como uno de sus postulados mejorar el acceso de toda la población a los servicios, como es el caso de la vacunación, por lo cual el aseguramiento es el mecanismo más importante que el gobierno ha implementado para disminuir las barreras económicas y las relacionadas con los servicios de salud identificadas previamente a la reforma.

En este contexto, el presente estudio examina la relación entre el status de aseguramiento y la cobertura de vacunación de niños y niñas menores de cinco años de edad provenientes de familias que viven en condiciones de alta vulnerabilidad social y que residen en asentamientos urbanos receptores de población desplazada por conflicto armado ubicados en los cuatro municipios que conforman el área metropolitana de Bucaramanga, capital del departamento de Santander, Colombia. 


\section{METODOLOGÍA}

Para recolectar los datos de este estudio se llevó a cabo una encuesta transversal, basada en la verificación del carné de vacunación de los niños y niñas menores de cinco años de edad provenientes de familias con alto nivel de vulnerabilidad social, las cuales comparten espacios geográficos y tienen características sociodemográficas y sanitarias similares y se diferencian por su condición de desplazamiento por conflicto armado. Las familias estudiadas estaban ubicadas en el principal asentamiento urbano, identificado como zona receptora de desplazados, de cada uno de los municipios que conforman el área metropolitana: Girón, Floridablanca, Piedecuesta y Bucaramanga. Todas las familias residentes en dichos asentamientos fueron elegibles para el estudio.

A través de un muestreo estratificado por municipio se seleccionaron los hogares desplazados. Para el cálculo de la muestra se consideró 50 \% de uso de servicios de vacunación, una precisión de 6 \% y un nivel de confianza de $95 \%$. La muestra de familias en situación de desplazamiento fue de 257. Para la población no desplazada se calculó un número igual de familias de manera que, por cada familia en desplazamiento ubicada en el asentamiento, se tuviera en cuenta una familia no desplazada del mismo asentamiento, obteniéndose así 514 familias a estudiar, las cuales se distribuyeron entre los municipios siguiendo el método de afijación proporcional (donde la fracción de muestreo fue diferente para cada uno de ellos).

Los datos fueron recabados mediante encuesta personal a la madre o al cuidador del niño, previo consentimiento informado por escrito, y todos los niños y niñas encontrados al momento de la encuesta sin esquema o con esquema incompleto de vacunación fueron remitidos a las instituciones responsables para su vacunación. La información sobre el estado de vacunación fue recolectada directamente del carné de vacunación que la madre o cuidador presentaba al encuestador. Todos los niños y niñas menores de cinco años de edad de la familia fueron incluidos para el estudio.

La variable dependiente fue definida como niño vacunado o no para la edad. Un niño fue clasificado como vacunado cuando de acuerdo con la edad había recibido todos los biológicos estipulados por el Programa de Inmunización del País (15). La variable independiente de interés fue status de afiliación al SGSSS. Los niños y niñas fueron clasificados en tres categorías: población vinculada al SGSSS, régimen subsidiado y régimen contributivo. Se consideró que un niño pertenecía a la primera categoría cuando no tenía ningún tipo de 
afiliación al SGSSS. En la segunda categoría se clasificaron aquellos niños y niñas afiliados a una Administradora de Régimen Subsidiado (ARS) a través de la asignación de subsidios del Estado y en la tercera categoría, cuando su familia mediante una cotización o prima estaba afiliada a una Empresa Promotora de Salud (EPS).

Otras variables independientes o covariables fueron estudiadas, las cuales incluyeron las características del niño, su familia y de los servicios de salud. Entre las características del niño se tuvo en cuenta la edad en meses o años cumplidos, sexo y asistencia al programa gubernamental de Atención a la Infancia del Instituto Colombiano de Bienestar Familiar (ICBF). Las características de la familia incluyeron: exposición o no al fenómeno del desplazamiento por conflicto armado; tiempo de residencia en el asentamiento (en meses); ingreso familiar; tamaño de la familia; historia previa de uso de servicios de salud; actitudes de la familia hacia la prevención, así como también el sexo, estado civil y escolaridad del jefe del hogar.

La familia fue clasificada como desplazada cuando presentaba el documento de la Red de Solidaridad Social (RSS), institución estatal, que la acreditaba como tal.

La muestra estudiada fue de 369 sujetos. Todas las variables nominales fueron descritas mediante porcentajes y las continuas mediante medidas de tendencia central y de dispersión, de acuerdo con su distribución. Para identificar la cobertura de vacunación y la asociación entre seguro de salud y estado de vacunación se estudiaron los niños y niñas cuyas madres o cuidadores presentaron el carné de vacunación $(n=170)$. Estos niños y niñas fueron clasificados en vacunados y no vacunados. Luego mediante regresión logística múltiple, y siguiendo los lineamientos de Hosmer y Lemeshow (16) se ajustó la relación entre el status de cobertura de seguro de salud y la condición de vacunación o no por las demás variables independientes. Un error $\alpha$ menor de 0.05 y un intervalo de confianza (IC) de $95 \%$ fueron definidos a priori como criterio de significancia. Al modelo final ingresaron las variables que presentaron un nivel de $p \leq 0.25$ en el análisis bivariado, y aquellas de relevancia teórica como ingreso, distancia a la institución de salud y actitud hacia la prevención.

Todos los cálculos se realizaron considerando a la familia como un conglomerado, de tal manera que se corrigiera el efecto de la posible correlación intraclase entre los niños y niñas que pertenecen a la misma familia. La bondad de ajuste del modelo fue evaluada mediante las pruebas de $\mathrm{x}^{2}$ de Pearson y de 
Hosmer-Lemeshow (16); la colinearidad de las variables fue evaluada con el factor de inflación de la varianza. Todos los análisis fueron desarrollados usando el programa estadístico Stata 8 (Stata Corporation, College Station, Texas).

\section{RESULTADOS}

La Tabla 1 describe las principales características sociodemográficas de los 369 niños y niñas. El 48,8 \% de los niños y niñas procedían de hogares en situación de desplazamiento por conflicto armado. Solamente un 22,8 \% de los niños y niñas estaban afiliados (asegurados) al SGSSS al momento de la encuesta. Entre los asegurados $77,4 \%$ lo estaba al régimen subsidiado, y el porcentaje restante al régimen contributivo.

Tabla 1. Características de los niños estudiados $(n=369)$ Situación de desplazamiento por conflicto armado (\%)

\begin{tabular}{|c|c|c|c|c|}
\hline & \multirow[b]{2}{*}{$n$} & \multicolumn{2}{|c|}{$\begin{array}{c}\text { Situación de } \\
\text { desplazamiento por } \\
\text { conflicto armado }(\%)\end{array}$} & \multirow[t]{2}{*}{$\begin{array}{l}\text { Valor } \\
\text { de } \rho\end{array}$} \\
\hline & & No & Si & \\
\hline Sexo & & & & 0.947 \\
\hline Mujer & 179 & 51,4 & 48,6 & \\
\hline Hombre & 190 & 51,1 & 48,9 & \\
\hline Edad (años) & & & & 0.054 \\
\hline Menos de uno & 62 & 43,6 & 56,4 & \\
\hline Uno & 80 & 48,8 & 51,2 & \\
\hline Dos & 78 & 59,0 & 41,0 & \\
\hline Tres & 78 & 42,3 & 57,7 & \\
\hline Cuatro & 71 & 62,0 & 38,0 & \\
\hline Status de afiliación al SGSSS & & & & 0.000 \\
\hline Vinculado (no asegurado) & 285 & 45,3 & 54,7 & \\
\hline Subsidiado & 65 & 67,7 & 32,3 & \\
\hline Contributivo & 19 & 84,2 & 15,8 & \\
\hline $\begin{array}{l}\text { Asistencia a programas } \\
\text { del ICBF }\end{array}$ & 292 & 51.0 & 49,0 & 0.870 \\
\hline $\begin{array}{l}\text { No } \\
\text { Si }\end{array}$ & 77 & 52,1 & 47,8 & \\
\hline Disponibilidad de carné & & & & 0.992 \\
\hline No & 199 & 51,2 & 48,8 & \\
\hline $\mathrm{Si}$ & 170 & 51,2 & 48,8 & \\
\hline \multicolumn{5}{|l|}{ Caracteristicas de la familia } \\
\hline Municipio & & & & 0.354 \\
\hline Bucaramanga & 232 & 49,6 & 50,4 & \\
\hline Floridablanca & 50 & 60,0 & 40,0 & \\
\hline Girón & 49 & 44,9 & 55,1 & \\
\hline Piedecuesta & 38 & 57,9 & 42,1 & \\
\hline Escolaridad del jefe de familia & & & & 0.011 \\
\hline (años) & 99 & 41,4 & 58,6 & \\
\hline Ninguno hasta 2 & 199 & 51,3 & 48,7 & \\
\hline $\begin{array}{l}\text { De } 2 \text { a } 5 \\
\text { Más de } 5\end{array}$ & 71 & 64,8 & 35,2 & \\
\hline
\end{tabular}


Aproximadamente la mitad de los niños y niñas vivían en hogares con ingreso diario familiar entre US\$ 0,36 a 1,78.

El jefe de la familia tenia en promedio 4,2 años de escolaridad y sólo 33,6 \% tenía primaria completa. Casi una cuarta parte de los hogares de los niños y niñas tardan en desplazarse 30 minutos o más en bus urbano a la institución pública de salud más cercana. Los hogares ubicados en el asentamiento de Bucaramanga y de Floridablanca eran los más cercanos, con un tiempo promedio en minutos de 5 y 10, respectivamente. Del total de niños y niñas estudiados, $46,1 \%(n=170)$ presentaron carné de vacunación al encuestador.

Cobertura de vacunación

El 22,9 \% de los niños y niñas que presentaron carné estaban vacunados para la edad, según el esquema de inmunización estipulado para Colombia. El porcentaje de vacunación, según edad, fue de $23 \%$ en menores de un año y 23,5 \% en los de 1 a 4 . En la Figura 1 se pueden observar las coberturas de vacunación por biológico y grupos de edad. En ella se nota que las mayores coberturas por biológicos las obtuvieron los niños y niñas de 24 y más meses de edad, y las vacunas de polio y antituberculosa (BCG) obtuvieron los porcentajes más altos, sin embargo, éstos no sobrepasaron el 60 y $70 \%$ respectivamente. $\mathrm{Al}$ contrario, la cobertura de haemophilus influenzae tipo b (HIb) fue la más baja en todas las edades.

Figura 1. Cobertura de Vacunación según edad y tipo de biológico

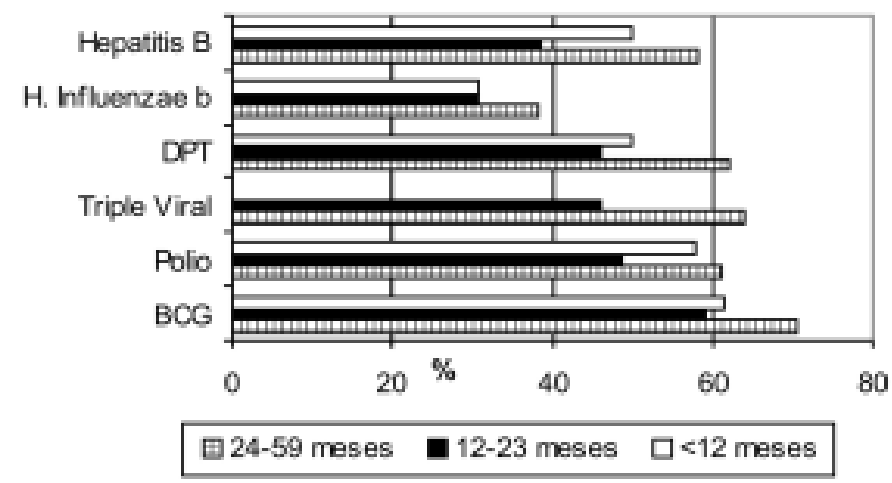


La cobertura de vacunación entre los niños y niñas afiliados al SGSSS ( $n=36$ ) fue de $30,5 \%$ comparado con $20,9 \%$ entre los no afiliados $(n=134)$ pero estas diferencias no fueron estadísticamente significativas, así como tampoco lo fueron las diferencias en la proporción de coberturas, según régimen de aseguramiento (datos no mostrados).

La cobertura de vacunación varió con otras características sociodemográficas tales como el tamaño del hogar y el nivel de escolaridad del jefe de familia; los niños y niñas cuyos hogares estaban conformados por más de cinco personas tuvieron mayor probabilidad de estar vacunados $(p<0.10)$, cuando se compararon con el grupo de referencia para tal característica y, por cada año que aumenta la escolaridad del jefe del hogar, la probabilidad de que el niño esté vacunado disminuye $(p<0.05)$ (efecto sin ajustar, datos no mostrados).

En la Tabla 2 se presenta el modelo de regresión logística múltiple; a este modelo ingresaron las variables que en el análisis bivariado presentaron una $p<0.25$ y aquellas que se consideraron de relevancia teórica como lo fueron ingreso y actitud de la familia hacia la prevención. De este modelo el status de seguro de salud fue predictor de estar vacunado para la edad $(p<0.05)$. Para los niños y niñas afiliados al régimen subsidiado la probabilidad de estar vacunados fue 2,4 veces más que la de los que no estaban afiliados al seguro de salud (vinculados al SGSSS).

Tabla 2. Asociación (Odds Ratios ajustados) entre el esquema completo de vacunación para la edad y sus potenciales determinantes

\begin{tabular}{lccc}
\hline \multicolumn{1}{c}{ Característica } & OR & IC 95\% & Valor $p$ \\
\hline $\begin{array}{l}\text { Familia desplazada por conflicto armado } \\
\text { No }\end{array}$ & 1,0 & & \\
Si & 1,8 & $0,79-4,09$ & 0.16 \\
Estado civil del jefe de familia & & & \\
$\quad$ Casado & 1,0 & & \\
Divorciado & 0,35 & $0,29-4,25$ & 0.41 \\
Soltero & 0,37 & $0,08-1,69$ & 0.20 \\
Unión libre & 0,59 & $0,18-1,92$ & 0.38 \\
Viudo & 1,34 & $0,94-19,2$ & 0.82 \\
Status de afiliación al SGSSS & & & \\
Vinculado (no afiliado) & 1,0 & & \\
Subsidiado & 2,4 & $1,03-5,59$ & 0.042 \\
Contributivo & 0,90 & $0,12-6,33$ & 0.92 \\
Escolaridad (años cursados) & 0,87 & $0,76-1,00$ & 0.063 \\
Actitud hacia la prevención & 0,69 & $0,24-1,95$ & 0.489 \\
Distancia a la institución de salud & 1,02 & $0,98-1,06$ & 0.267 \\
\hline
\end{tabular}




\section{DISCUSIÓN}

Nuestro estudio permitió determinar muy bajas tasas de afiliación al SGSSS y de cobertura de esquema completo de vacunación para la edad. De igual forma, ninguno de los biológicos presentó coberturas útiles. Estas coberturas difieren de las encontradas en estudios realizados en población infantil de distintos estratos socioeconómicos en Colombia(3,7,9), y en otros países $(14,17)$ y son muy bajas comparadas con las observadas a nivel de cada uno de los municipios estudiados y con las coberturas obtenidas para el Departamento de Santander en el mismo año del estudio (18).

Sin embargo, nuestros resultados son consistentes con los que otros autores han encontrado respecto al uso de servicios de vacunación en población asegurada, pobre y urbana (14), así como las coberturas de inmunización en poblaciones desplazadas y no desplazadas ubicadas en zonas receptoras de desplazamiento (19), y en municipios que presentan una alta proporción de familias desplazadas (8), lo cual vislumbra fallas en las políticas de ampliación de cobertura y en el funcionamiento y prestación del programa de inmunización en contextos complejos de pobreza y desplazamiento. Con esta investigación también fue posible establecer bajas coberturas por biológicos en todas los grupos de edad, lo que refleja que los problemas de acceso a las vacunas para esta población no sólo se presentan en el año previo al estudio, sino que se han mantenido por varios años, es decir, muestra una situación previa de barrera a los servicios de vacunación.

Estar afiliado al SGSSS, a través del régimen subsidiado, aumentó la probabilidad de estar vacunado, en comparación con los no afiliados. Otros estudios en Colombia han mostrado que la afiliación está asociada a la vacunación $(3,7,13)$, aunque en uno de ellos se encontró que el régimen contributivo aumenta la probabilidad de estar vacunado (7). Por el contrario, otros reportes de investigación han mostrado que estar asegurado no afecta la probabilidad de recibir vacunas, aun después de haber ajustado por variables sociodemográficas y de los sistemas de salud $(12,20)$.

Ninguna de las covariables sociodemográficas que se estudiaron y por las cuales se ajustó la relación entre aseguramiento y estar vacunado resultó asociada a la vacunación, lo cual está en desacuerdo con otros estudios de vacunación infantil, en donde la edad del niño, el nivel educativo del jefe del hogar y el tamaño de la familia, han resultado asociados en población pobre colombiana, y con resultados de otros estudios realizados en contextos distintos 
al colombiano (12). Este hecho también fue encontrado al estudiar el uso de servicios médicos por población desplazada, en donde los factores tales como edad, sexo, ingreso y nivel educativo no resultaron asociados al uso de servicios por enfermedad(21). El que ninguna de las covariables hubiese resultado asociada podría estar sugiriendo que otros factores como los relacionados con el sistema de aseguramiento y el funcionamiento de la red de prestadores de las entidades aseguradoras de salud, son responsables de las bajas coberturas de uso de servicios de salud en este tipo de población.

De hecho, diversos estudios en poblaciones pobres urbanas $(9,21)$ han mostrado que las características de la población, incluyendo las actitudes y las creencias, no explican totalmente el uso del servicio de vacunación pero si hacen énfasis en las características del sistema de prestación de servicios como variable explicatoria importante. De igual manera, otros trabajos muestran cómo las coberturas de vacunación están fuertemente influenciadas por el comportamiento de los proveedores (23-25).

Distintas estrategias existen para medir la cobertura de vacunación (26), y múltiples polémicas se presentan en el análisis de cobertura de vacunación a nivel institucional y poblacional, debido a las limitaciones en la información relacionada, en el primero, con las estimaciones de población para los diversos grupos de edad y, en el segundo, con la precisión del autoreporte de la madre. Nosotros decidimos indagar el estado de vacunación de los niños y niñas a partir de la revisión del carné. Este hecho pudo haber introducido un sesgo de selección que resultara en subestimación de la cobertura pues muchos de los niños y niñas que no disponían de carné podrían haber tenido el esquema completo de vacunación para la edad. Sin embargo, se puede descartar la presencia del sesgo de selección en nuestro estudio porque al comparar el grupo de niños y niñas que contaba con carné con el que no disponían de él, no se observaron diferencias estadísticamente significativas en las variables que se midieron y que se han encontrado asociadas con status de vacunación en otros estudios, incluyendo las que quedaron en nuestro modelo final.

Varios trabajos en Colombia han demostrado una pérdida del carné entre 25 a 49 \% (1). A pesar de que nuestro estudio demuestra un porcentaje bajo de disponibilidad de carné, consideramos que éste era el mejor registro para indagar, en forma confiable, la cobertura de vacunación en dicha población. Un estudio previo a la realización de la encuesta mostró que 70 \% de las madres, desplazadas y no desplazadas, ubicadas en el asentamiento del Café Madrid, de Bucaramanga, no tenía información adecuada sobre el esquema de las vacunas de sus hijos(27). 
A pesar de las limitaciones propias de los estudios transversales (28) en los cuales es difícil explorar aspectos que permitan entender cómo opera el aseguramiento sobre la cobertura de vacunación, el aporte más importante de esta investigación fue mostrar que el sistema de aseguramiento no está funcionando para proteger contra enfermedades inmunoprevenibles a poblaciones con severas carencias materiales y problemas sociales. Aproximadamente una tercera parte de los niños y niñas afiliados al régimen subsidiado estaban vacunados para la edad, mientras que en el régimen contributivo sólo uno de cada cinco lo estaba.

Los hallazgos de esta investigación sugieren que existen barreras para que esta población acceda a los servicios de vacunación y proveen información para que las autoridades de las Direcciones Locales de Salud de cada municipio y los proveedores del servicio de vacunación promuevan y restablezcan la cobertura de vacunación a partir de la focalización de acciones que la mejoren. Por ejemplo, la oferta de vacunación extramural y la canalización hacia el servicio, así como un mayor monitoreo y supervisión, son algunas de las estrategias que mejoran la cobertura de inmunización infantil $(29,30)$.

La cobertura de vacunación es uno de los indicadores más comúnmente usados para referirse a la calidad de la atención en salud entre los niños y niñas, y es una intervención altamente costo-efectiva para disminuir la carga de la enfermedad en la población infantil. Las autoridades de salud de los cuatro municipios donde se ubican los asentamientos deben reestructurar las políticas de aseguramiento y de monitoreo del desempeño de los servicios de inmunización para la población estudiada •

Agradecimientos. Esta investigación fue financiada y realizada por la Universidad Industrial de Santander (UIS) y la Organización Panamericana de la Salud (OPS). La responsabilidad de los resultados que aquí se presentan es exclusivamente de las autoras.

\section{REFERENCIAS}

1. Profamilia. Salud sexual y reproductiva en Colombia. Encuesta nacional de demografía y salud 2000. Santafé de Bogotá: Profamilia; 2000. pp: 122-126.

2. Profamilia. Salud sexual y reproductiva en Colombia. Encuesta nacional de demografía y salud 2005. Santafé de Bogotá: Profamilia; 2005. pp:222-29.

3. Tono T, Velásquez L, Castañeda L. El impacto de la reforma sobre la salud pública. El caso de la salud sexual y reproductiva. Santa Fe de Bogotá: Fundación Corona, Engender Health Foundation Ford; 2002. 
4. Homedes N, Ugalde A. Las reformas de salud neoliberales en América Latina: una visión crítica a través de dos estudios de caso. Rev Panam Salud Publica/Pan Am J Public Health 2005;17(3): 210-20.

5. Organización Panamericana de la Salud, Ministerio de Protección Social. Programa de inmunizaciones.Inf Quinc Epid Nac, 2000;6(8):114-24.

6. Ayala-cerna C, Kroeger A. La reforma del sector salud en Colombia y sus efectos en los programas de control de tuberculosis e inmunización. Cadernos de Saúde Pública 2002; 18(6):1771-81.

7. Prieto F, De la hoz F. Evaluación de la jornada nacional de vacunación en Bogotá D.C. Rev Salud Pública 2003;5(2):144-57.

8. Rojas J, Prieto F. Impacto de la jornada nacional de vacunación contra la poliomielitis en Colombia, 2001: una aproximación ecológica. Rev Salud Pública. 2004;6(1):1-17.

9. Acosta N, Rodríguez J. Inequidad en las coberturas de vacunación infantil en Colombia, años 2000 y 2003. Rev Salud Pública. 2006; Sup 8(1):102-15.

10. Newacheck PW, Hughes DC, Stoddard JJ. Children's access to primary care: Differences by race, income, and insurance status. Pediatrics 1996;97:26-32.

11. Holl JL, Szilagyi PG, Rodewald LE, Byrd RS, Weitzman ML. Profile of uninsured children in the United States. Arch Pediatr Adolesc Med 1995;149:398 -406.

12. Santoli JM, Huet NJ, Smith PJ, Barker LE, Rodewald LE, Inkelas M, Olson LM, Halfon $\mathrm{N}$. Insurance status and vaccination coverage among US preschool children. Pediatrics 2004;113(6):1959-64.

13. Cruz LF, Girón LN, Velásquez R, García LM, AlzateA. Coberturas de vacunación en el Valle del Cauca, 2002. Colombia Médica 2003;34(1):17-23.

14. Acosta-Ramírez N; Durán-Arenas LG;Eslava-Rincón JL; Campuzano-Rincón JC. Determinants of vaccination of vaccination after the Colombian health systems reflrm. Rev Saude Publica 2005;39(3):421-29.

15. Ministerio de Salud de Colombia. Resolución 412 de 2000. Norma Técnica para la vacunación según el Programa Ampliado de Inmunizaciones, PAI.

16. Hosmer D, Lemeshow S. Applied logistic regression. Segunda edición, New York: John Wiley \& Sons, INC; 2000.

17. Zimmeerman R, Medgsger A, Ricci R, Raymund MM, Mieczkowski T, Grufferman S. Impact of free vaccine and insurance status on physician referral of children to public vaccine clinics. JAMA 1997;278(12):996-1000.

18. Secretaria de Salud de Santander. Indicadores Básicos de Salud; 2003.

19. Organización Panamericana de la Salud. Serie salud y desplazamiento en Colombia. Módulo 1. Bogotá: Universidad de Antioquia; 2005.

20. Alessandrini EA, Shaw KN, Bilker WB, Schwaez DF, Bell LM. Effects of medicaid managed care on quality: Childhood inmunizations. Pediatrics 2001;107: 133542 
21. Ruiz-Rodríguez M, López-Moreno S, Ávila-Burgos L, Acosta-Ramírez N. Utilización de servicios médicos por una población desplazada en Bucaramanga.Rev. Salud Pública 2006;8(3):197-206.

22. Brenner RA, Simons-Morton BG, Bhaskar B, Das A, Clemens JD. Prevalence and Predictors of Immunizations among Inner-city Infants: a Birth Cohort Study. Pediatrics 2001;108(3):661-70.

23. Santoli JM, Szilagyi PG Rodewald LE. Barriers to immunization and missed opportunities. PediatrAnn.1998;27(6):366-74.

24.Briss PA, Rodewald LE, Hinman AR, et al. Reviews of evidence regarding interventions to improve vaccination coverage in children, adolescents, and adults. Am Prev Me.2000;18(suppl):97-140.

25. Szilagyi PG, Vann J, Bordley C et al. Interventions aimed at improving immunization rates. Cochrane Database Syst Rev 2002;(4):CD003941.

26. Murray CJL, Shengelia B, Gupta N, Moussavi S, Tandon A, Thieren M. Validty of reported vaccination coverage in 45 countries. The Lancet 2003;362:1022-27.

27. Ruiz-Rodríguez M, Angulo-de Meza ML, Castillo E, Fernández CG García J, Gómez CF. Conocimientos de las madres sobre los programas de prevención de la enfermedad y promoción de la salud para la población infantil. Café Madrid, Bucaramanga: Universidad Industrial de Santander.UIS; 2001.

28. Hernández B, Velasco-Mondragón HE. Encuestas transversales. Salud Pública de México 2000;42(5):447-55.

29. Pegurri E, Fox-Rushby JA, Damián W. The effects and costs of expanding the coverage of inmunization services in developing countries: A systematic literature review. Vaccine 2005;23:1624-35.

30. Committee on community health services and committee on practice and ambulatory medicine. Increasing inmunization coverage. Pediatrics 2003;112:993-96. 